\author{
Questions Questions Vives \\ vives \\ Recherches en éducation \\ $N^{\circ} 23 \mid 2015$ \\ Évaluation et changement
}

\title{
Évaluation et changement
}

\section{Yvan Abernot}

\section{(2) OpenEdition}

\section{Journals}

\section{Édition électronique}

URL : http://journals.openedition.org/questionsvives/1704

DOI : 10.4000/questionsvives. 1704

ISSN : 1775-433X

\section{Éditeur}

Université Aix-Marseille (AMU)

\section{Édition imprimée}

ISBN : 978-2-912643-47-6

ISSN : 1635-4079

\section{Référence électronique}

Yvan Abernot, «Évaluation et changement », Questions Vives [En ligne], № 23 | 2015, mis en ligne le, consulté le 22 septembre 2020. URL : http://journals.openedition.org/questionsvives/1704 ; DOI : https://doi.org/10.4000/questionsvives.1704

Ce document a été généré automatiquement le 22 septembre 2020.

\section{(c) (i) (9)}

Questions Vives est mis à disposition selon les termes de la licence Creative Commons Attribution Pas d'Utilisation Commerciale - Pas de Modification 4.0 International. 


\title{
Évaluation et changement
}

\author{
Yvan Abernot
}

1 Les travaux exposés ci-dessous ont été présentés lors des Journées Internationales de l'UMR EFTS (Education Formation Travail Savoirs) de Toulouse en novembre 2014. Le symposium qui a réuni les chercheurs impliqués leur a donné l'opportunité de discuter et d'améliorer leur production. Cette prise de connaissance réciproque des écrits des participants a permis à chacun de se situer, ce qui facilite l'éditorial qui suit.

2 L'évaluation a toujours servi à donner des informations, c'est sa fonction. Pourtant, dans ce numéro consacré à ses évolutions, il va apparaître que ses éléments constitutifs ou associés sont en mouvement. En reprenant une organisation classique : évaluateur, action, nature, fonction, forme, objet, résultat, destinataire, répercussion (concepts tous entendus au singulier ou au pluriel), les contributeurs vont mettre au jour de nombreux déplacements. L'évaluation donne, certes, toujours des informations mais elle est aussi de plus en plus outil central de formation. Il ne s'agit pas seulement d'évoquer l'évaluation formative qui elle aussi prend des formes nouvelles d'applications (Mottier, ci-après), mais par exemple, de considérer le « connais-toi toimême » comme finalité formative première, appuyée sur l'évaluation. Lors d'une VAE, par exemple (cf. Eymery, ci-après), le principal travail consiste à faire évoluer le candidat en partant de ce qu'il sait faire pour le faire évoluer vers ce qu'il sait dire de ce qu'il sait faire.

3 Il a toujours été conçu que l'on pouvait apprendre sur soi à partir de l'évaluation mais jusqu'alors, il s'agissait de connaître la valeur du travail produit. Voilà que l'on peut aussi, en apprendre sur soi-même. En évaluation formative aussi, le rendu à l'élève le fait évoluer mais ce n'est qu'à long terme car l'objectif premier est de faire améliorer le produit du travail de l'élève. Le but s'est donc déplacé tout en conservant le principe d'un changement attendu grâce à l'évaluation. L'évolution de la personne est visée d'emblée.

4 Catherine Eymery montre ce qui change vraiment chez les sujets de son investigation : la tolérance à l'ambiguïté, la croyance en l'origine du succès, la flexibilité et le type de motivation. Une évolution sur une seule variable change le système d'attitudes de la 
personne. Il serait donc bien réducteur de confondre la VAE avec une simple attestation de savoir-faire.

5 Cette nouvelle forme d'évaluation montre une ambition individuelle et sociale que ne manquent pas de relever aussi Véronique Bedin et Yvan Abernot dans leur texte introductif. Il est instructif de constater qu'épistémologiquement, évaluation et changement sont nécessairement liés puisque la première ambitionne de dire les états et les évolutions, pendant que le deuxième ne se détecte que sur la base de critères (explicites ou non).

6 En prenant le parti d'évaluer le changement par le biais des évaluations, le premier constat est celui d'une inflation. Quelle est cette société qui autorise et interdit sur la base de brevets, certificats, diplômes, permis, etc. ? Au-delà des questions de validité docimologique, un des corollaires de ces sanctions est la formation. De ce point de vue, les certifications peuvent être positivement envisagées puisqu'elles participent à de multiples formations. Mais un autre corollaire est le cadrage de l'activité. Ainsi, par exemple, l'évaluation des enseignants chercheurs universitaires organise dorénavant leur travail et occupe une part toujours plus importante de leur temps en constitution de dossiers. Des tableaux de bonnes pratiques se font jour dans tous les milieux. Ils n'ont pas seulement le rôle fantasmatique du contrôle généralisé mais aussi celui de rapprocher l'action humaine d'un modèle mécanique donc à terme de les rendre substituables. Ainsi, les bornes de péage, les pilotes automatiques, les Massive Open Online Courses, etc. remplacent les personnes.

7 Ces deux tendances se dégagent très bien de l'article de Lucie Aussel et Jean-François Marcel : une évaluation participant à l'évolution de la personne, une évaluation qui vise à modéliser les activités et les formations qui y sont associées.

Véronique Bedin et Yvan Abernot se dotent d'un outil conceptuel d'évaluation du changement qui permet d'abord l'établissement d'un état dont il faudra sélectionner des facettes pour les voir évoluer. Ainsi définie, l'évaluation s'étend aux objets physiques, aux individus, aux organisations, aux sociétés. Il est alors possible de rendre compte de changements par rapport à un objet ou un repère fixes. Dans d'autres cas, se sont les cadres qui évoluent, rendant les objets différents, non en eux-mêmes mais relativement à d'autres. Ainsi, ce qui est nouveau ne le reste pas. C'est le contexte qui évolue. Le changement est aussi celui du regard.

Dans cette perspective, un certain nombre d'évolutions de l'évaluation scolaire sont analysées dans leur temporalité et leur contexte. Par exemple, depuis les années 2010, le principe même de notation est remis en question. Ce n'est pas la première fois puisque après 1968, une échelle ordinale inspirée de celle des anglo-saxons avait remplacé provisoirement la sacrosainte échelle de 0 à 20 . Mais comme nous l'avons tous constaté : elle est très vite revenue. Pendant que la notation gagne du terrain vers la maternelle, certains établissements du second degré la suppriment (pour expérimentation). Dans le secondaire, l'évaluation par compétences donne bien du mal aux enseignants de bonne volonté et dans l'enseignement supérieur, le LMD généralise le cursus des études en Europe, dans la perspective d'une mobilité en principe, facilitée ! En fait de simplification, le parcours qui mène au pays d'accueil est tellement semé d'embûches que peu d'étudiants le pratiquent avec succès. Mais surtout, de quels étudiants s'agit-il ? Majoritairement des jeunes que la mobilité n'effraie pas et qui, de surcroît, résistent au parcours initiatique administratif. S'il y a changement, ce n'est pas du point de vue démocratique, c'est plutôt, comme indiqué plus haut, du fait que la 
formation de la personne domine les considérations de savoirs bruts. Or, les compagnons du devoir nous l'ont appris, c'est le chemin qui est formatif.

À l'université, ce qui occupe de plus en plus de professionnels, ce sont les dossiers. Dossiers de CNU, dossiers HCERES, dossiers d'accréditation, dossiers ANR, etc. La tendance "contrôle généralisé " s'y développe en gagnant sur les fonctions d'enseignement et surtout de recherche.

Quant à la tendance formation de la personne, concernant les enseignants, elle ne les concerne que très peu. Quand ils pourraient, par exemple, bénéficier de l'évaluation formative issue des retours des étudiants, c'est l'administration qui l'impose et l'exploite!

12 Parmi d'autres "innovations", les auteurs analysent la pédagogie inversée et la formation à distance. Elles ne visent pas le développement des étudiants, elles tablent sur leurs forces initiales et éliminent rapidement les plus en difficulté, non seulement avec les savoirs académiques mais surtout avec eux-mêmes. La pédagogie inversée concerne des étudiants (ou des élèves) déjà assez «solides " pour travailler le cours avant que le formateur entre en situation. Ces étudiants existent bel et bien : dans les grandes écoles. De la même manière, toutes les formes d'enseignement à distance supposent que l'apprenant s'engage, travaille régulièrement toute l'année et produise les synthèses nécessaires aux examens ... comme si tous en étaient capables. Et tous les forums, aides individualisées, facilités d'accès au "savoir universel » etc. ne changent rien aux résultats peu encourageants qui ne responsabilisent l'apprenant que dans son échec. L'aspect sélectif est indéniable (contrairement à l'affichage) mais une fois encore, c'est la formation de la personne qui est visée. Ceux qui réussissent dans ces conditions en sortent certes plus savants mais surtout plus forts. Ils ont consolidé leur rapport au savoir.

teurs développent aussi le rapport de l'évaluation aux changements de missions de l'université. La préoccupation d'employabilité impose de nouvelles façons de former et d'évaluer. Si les diplômes ont toujours été suspectés par les employeurs, leur absence reste rédhibitoire!

Mais la proximité de l'université avec l'entreprise se repère aussi dans l'évolution de la recherche. Ainsi, Lucie Mottier montre comment des recherche-actions affermissent leurs statuts, surtout quand d'autres formes de rapport ont échoué. Il ne s'agit plus de faire de l'application des données de recherche au monde du travail mais de développer l'esprit de recherche avec les praticiens.

15 Bedin et Abernot montrent aussi le désarroi d'une institution critiquée par tous ses partenaires, de l'intérieur comme de l'extérieur. Sur la base d'une enquête d'une centaine de personnes comprenant des élèves, des enseignants et des parents, ils relèvent les critiques les plus souvent portées des uns sur les autres et sur l'École en général. En dehors de l'évocation courante d'un passé idéal, le seul point commun est l'inadaptation. La reconnaissance due à une École ayant accompagné l'accession des citoyens à la République semble s'éloigner en ce début de siècle où l'influence d'un système de formation sur une société en devenir s'oppose à la pression d'un monde marchant qui exige que ses futurs contributeurs correspondent à ses besoins, voire à ses valeurs.

16 Avec Lucie Mottier, beaucoup de ceux qui s'intéressent à l'évaluation formative ont vu le concept puis les tentatives d'application se développer dans les dernières décennies 
du vingtième siècle. Nous avons également vu, en Suisse où elle travaille, comme en France et ailleurs, l'élan faiblir, voire les textes réglementaires gommer les mentions à ce concept. Elle pose donc explicitement sur la base d'une analyse rigoureuse et exhaustive, la question de l'adoption des propositions scientifiques fondées sur les travaux de recherche universitaire, leur développement et leur abandon. Il aurait pu s'agir de la lecture globale ou des mathématiques modernes. Certaines innovations portant parfois des enjeux lourds sont dans un triptyque vertigineux: chercheurs, politiques, praticiens!

Mais l'abîme n'est pas inéluctable. En s'appuyant sur trois expériences de rechercheaction, Lucie Mottier montre que l'on peut faire évoluer les pratiques quand les propositions émergent du groupe de travail. Les praticiens vont ensuite les adapter à leur contexte et se les approprier comme on façonne un outil à sa main. Il est donc possible de promouvoir l'évaluation formative mais sans l'imposer voire même, sans la nommer!

Lucie Aussel et Jean-François Marcel confirment la même tendance à moins dissocier les acteurs catégoriels, ici chercheurs et actifs des entreprises. Ils rendent compte d'une recherche-intervention où le premier travail consiste à définir l'objet d'évaluation. Or il est double: donner des informations pour objectiver et des informations pour expliquer. La commande fait toujours l'objet d'une analyse voire d'une négociation, car si l'objet était bien défini d'emblée, il n'y aurait pratiquement pas besoin de chercheur pour l'évaluer. Leur travail essentiel consiste précisément à définir quoi regarder.

Plusieurs centaines d'étudiants de BTSA entrent en expérimentation dans un dispositif destiné à améliorer leur insertion professionnelle. Sans entrer ici dans les détails, cette recherche montre bien comment ce que nous appelions les "éléments associés » à l'évaluation au début de cet éditorial, relèvent des deux tendances évoquées ci-dessus. Nous l'avons vu, l'objet est double. En revanche, les observés sont dans ce cas aussi destinataires puisque dès le début ils travaillent ensemble au «montage du projet». C'est ainsi qu'il est escompté que les étudiants trouvent plus vite du travail (variable officielle) parce qu'ils auront acquis certaines compétences de par leur participation au projet (variable explicative complémentaire ou sous-jacente aux activités originales proposées).

Pour conclure ces quelques pages, je dirai ma satisfaction de voir toujours se développer la réflexion, mais aussi de plus en plus, la mise en pratique des idées porteuses de progrès pédagogiques, donc sociaux. J'en remercie les contributeurs de ce numéro thématique " Évaluation et changement ».

\section{AUTEUR}

\section{YVAN ABERNOT}

Professeur à l'ENFA de Toulouse (École Nationale de Formation Agronomique) 